\title{
Protective Role of Panax Notoginseng Saponins in Hydrocortisone-Induced Injury in HBMECs by Promoting APOA1 Expression
}

\section{Zejian Jin}

Tongliao City Hospital

Fei Yan ( $\square$ yanfei_fyanf@163.com )

Tongliao City Hospital https://orcid.org/0000-0001-8655-4159

\section{Hetian Song}

Tongliao City Hospital

\section{Yonggang Wang}

Tongliao City Hospital

\section{Shun Bai}

Tongliao City Hospital

\section{Zhongyu Lan}

Tongliao City Hospital

\section{Bin Liu}

Tongliao City Hospital

\section{Lei Miao}

Tongliao City Hospital

\section{Research article}

Keywords: panax notoginseng saponins (PNS), human bone microvascular endothelial cells (HBMECs), APOA1

Posted Date: April 28th, 2020

DOI: https://doi.org/10.21203/rs.3.rs-23611/v1

License: (c) (i) This work is licensed under a Creative Commons Attribution 4.0 International License. Read Full License 


\section{Abstract \\ Background}

To evaluate the protective effect and underlying mechanism of panax notoginseng saponins (PNS) on hydrocortisone (HC)-induced injury in human bone microvascular endothelial cells (HBMECs).HBMECs were isolated from femoral heads resected in total hip arthroplasty and identified by morphology and immunofluorescence.

\section{Methods}

HBMECs were injured by hydrocortisone $(\mathrm{HC})$, treated with PNS in different concentrations, and transfected with APOA1 or siAPOA1 to explore the role of APOA1 in the process where PNS protected HCinjured HBMECs. The viability and apoptotic rate were determined by CCK-8 and flow cytometry assays, respectively. The migration and tube length of HBMECs were evaluated by wound healing and tube formation assays, respectively. The mRNA expression of APOA1 was determined by qRT-PCR. The proteins levels of APOA1, Bcl-2, Bax and Caspase-3 were determined by Western blot.

\section{Results}

HBMECs exhibited spindle-shaped morphology. CD31 and vWF served as positive control and CD133 served as negative control. The viability of HBMCS inhibited by HC was balanced with the lower mRNA expression of APOA1 in a dose-dependent manner. PNS reversed the inhibitory effect of $\mathrm{HC}$ on the mRNA expression of APOP1, cell viability, migration, tube length, and the stimulative effect of $\mathrm{HC}$ on apoptosis of HBMECs. APOA1 overexpression further promoted the protective effect of PNS on HC-inhibited cell viability, migration and tube length, and $\mathrm{HC}$-promoted apoptosis, whereas APOA1 silencing inhibited the protective effect of PNS and further promoted the adverse effect of HC on HBMECs.

\section{Conclusion}

PNS can efficiently suppress HC-induced injury in HBMECs, which may be associated with over-expressed APOA1.

\section{Introduction}

Osteonecrosis of the femoral head (ONFH) most frequently comes from trauma or the use of corticosteroid and alcohol, while it is also related to blood dyscrasias and metabolic and coagulation disorders (1). Among the above factors, corticosteroid use is the most common cause of ONFH (2). Because treatment with glucocorticoids (one kind of corticosteroid) results in reduced blood flow and hypercoagulability, glucocorticoids damage the bone microvascular endothelial cells (BMECs) of the 
femoral head (3). BMECs form monolayer junctions attached to bone trabeculae, which are in direct contact with the ingredient in the blood to provide oxygen and nutrition for bone tissues and cells (4). BMECs injury and dysfunction in femoral head are believed to play a significant role in the pathogenesis of femoral head necrosis (5). However, because of the complex etiology, the exact mechanisms of ONFH pathogenesis are not completely clear, and treatment with good long-term outcomes is yet to be developed.

The major apolipoproteins of plasma lipoproteins generally play important parts in preserving the structural integrity of lipoprotein particles and in physiological functions of lipoproteins (6).

Apolipoprotein A1 (APOA1), one type of apolipoprotein A family, is a primary protein component of highdensity lipoprotein (HDL) (7), and plays a role in reverse cholesterol transport that initiates multiple cellular pathways by binding to its receptor ABCA1 (8). Wu, $\mathrm{X}$ et al. (9) reported that down-expressed APOA1 improves risk prediction of Type-2 diabetes mellitus. Tuft Stavnes, $\mathrm{H}$ et al. reported that mRNA expression of APOA1 is a marker of longer survival in ovarian serous carcinoma effusions (10). However, the function of APOA1 in BMECs is still not clarified yet.

Developed in ancient China, Traditional Chinese Medicine provides comprehensive holistic therapies and has been confirmed useful in the prevention and therapy of most pathologies worldwide (11). Notoginseng, predominantly cultivated in China and Japan, is defined in Chinese medicine as warm in nature (12). Panax notoginseng saponins (PNS) come from the active component of panax notoginseng root and can relieve swelling, promote blood clotting and alleviate pain with many biological activities such as immunomodulatory effects, antioxidation and anticancer properties (13). However, the effect of PNS on glucocorticoids-induced injury in human bone microvascular endothelial cells (HBMECs) remains to be elucidated.

In the present study, HBMECs were isolated from human femoral heads and used to investigate the effect of PNS on hydrocortisone (HC)-induced injury in HBMECs. To further elucidate the potential mechanism of this effect, we explore the role of APOA1 in the protective effect of PNS on HC-induced injury in HBMECs.

\section{Materials And Methods}

\section{BMECs isolation, culture and identification}

The human femoral head samples were collected from voluntary patients who underwent total hip arthroplasty at Tongliao City Hospital between 03/22/2018 and 03/22/2019 due to femoral neck fracture, primary hip osteoarthritis or osteoarthritis secondary to congenital hip dysplasia. This research was approved by the Ethics Committee of the Tongliao City Hospital. Written informed consents from all patients were obtained in any experimental work with humans.

Under aseptic condition, HBMECs were isolated from the femoral head samples and cultured with DMEM as described previously (14). Seven days after the culture, the morphology of the isolated HBMECs was 
observed by phase-contrast microscope. The expression levels of the markers CD31 (PA5-16301), CD133 (PA5-38014) and von Willebrand factor (vWF, PA5-16634) (Thermo Fisher Scientific) were determined by immunofluorescence according to previous report $(14,15)$.

\section{Cell Treatment}

HBMECs were cultured with $5 \% \mathrm{CO}_{2}$ at $37^{\circ} \mathrm{C}$ with different concentrations $(0.25,0.5,0.75$, or $1.0 \mathrm{mg} / \mathrm{ml})$ of $\mathrm{HC}$ (386698, Sigma) for $24 \mathrm{~h}$ to establish the HC-induced cell model (16). As shown in Fig. 2A, because $0.759 \mathrm{mg} / \mathrm{ml}$ was the IC50 of $\mathrm{HC}, 0.75 \mathrm{mg} / \mathrm{ml}$ of $\mathrm{HC}$ was used for next experiments.

To test the effect of PNS (CP201608022, Yunnan phytopharmaceutical. Co., Ltd. Yunnan, China) on cell viability, HBMECs were cultured with PNS for $24 \mathrm{~h}$ in different concentrations $(100,200,300,400 \mathrm{mg} / \mathrm{ml})$.

To test the protective effect of PNS on HC-induced cell injury, HBMECs were co-cultivated with PNS in different final concentrations $(100,200,300,400 \mathrm{mg} / \mathrm{ml})$ and $\mathrm{HC}$ in final concentration $(0.75 \mathrm{mg} / \mathrm{ml})$ for $24 \mathrm{~h}$.

To test whether APOA1 participated in the protective effect of PNS on HBMECs exposed to HC, BMECs were transfected with APOA1 or siAPOA1 to up-regulate or down-regulate the expression of APOA1.

\section{HBMECs Transfection}

The pcDNA3.1 APOA1, pcDNA3.1, APOA1 siRNA (siAPOA1) and siRNA negative control (siNC) were obtained from RiboBio (Guangzhou, China).

HBMECs transfected with pcDNA3.1 was served as negative control (NC) of pcDNA3.1-APOA1. HBMECs transfected with siNC was served as negative control of siAPOA1. Before harvest, the vectors were transfected into HBMECs for $48 \mathrm{~h}$ to down-regulate the expression of APOA1 in HBMECs using Lipofectamine ${ }^{\circledR} 2000$ Transfection Reagent (11668, ThermoFisher, USA).

\section{CCK-8 Assay}

HBMECs $\left(5 \times 10^{4}\right.$ cells/well) were planted in 96 -well plates for $24 \mathrm{~h}$. After $24 \mathrm{~h}$ indicated treatment, the viability of HBMECs was determined by CCK-8 kit (CA1210, Solarbio, China) and the absorbance was determined at $450 \mathrm{~nm}$ with a microplate reader (SpectraMax iD5, Molecular Devices, US).

\section{Reverse Transcription And Quantitative Real-time PCR}

Total RNA of HBMECs was extracted by Trizol reagent (15596018, Invitrogen). PrimeScript ${ }^{\text {TM }}$ RT Master Mix was used for RT-PCR (RR036B, Takara). QRT-PCR was performed by 7300 real-time PCR system 
(Applied Biosystems, USA) and TB Green ${ }^{\circledR}$ Premix Ex Taq ${ }^{\mathrm{TM}}$ II (RR820Q, Takara). Conditions were as follows: 30 min of incubation at $95^{\circ} \mathrm{C}$, followed by amplification of $5 \mathrm{sec}$ at $95^{\circ} \mathrm{C}, 34 \mathrm{sec}$ at $60^{\circ} \mathrm{C}$ for 40 cycles. Data were analyzed by the $2^{-\Delta \Delta C t}$ method (17). The sequences of primers used in this research were displayed in Table 1.

Table 1

Primer sequences used for quantitative real-time reverse transcription polymerase chain reaction (qRT-PCR)

\begin{tabular}{|lll|}
\hline Genes & & Primer sequences $\left(\mathbf{5}^{\mathbf{}^{\prime} \mathbf{3}^{\prime} \mathbf{)}}\right.$ \\
\hline FOXF1 & forward & CAAAGACAGCGGCAGAGACTAT \\
& reverse & CTGTCTCCTTTTCCAGGTTATCC \\
GADPH & forward & CCAGGTGGTCTCCTCTGA \\
& reverse & GCTGTAGCCAAATCGTTGT \\
\hline
\end{tabular}

\section{Western Blot}

HBMECs lysates were lysed for protein isolation and analyzed as previously described (16). In brief, HBMECs lysates were prepared with RIPA buffer (P0013, Beyotime, China) containing protease inhibitors. After protein containing compounds was centrifuged by high speed centrifuge $(12,000 \mathrm{rpm}, 5 \mathrm{~min})$ at $4{ }^{\circ} \mathrm{C}$, the supernatant was obtained. The total protein concentration was determined by double bicinchoninic Acid (BCA) assay kit (P0009, Beyotime, China). The protein was separated by $8 \%$ SDS-PAGE gel and transferred to a PVDF membrane (FFP24, Beyotime) at $4{ }^{\circ} \mathrm{C}$ for $2 \mathrm{~h}$. Then, $5 \%$ skimmed milk was prepared using Tris-buffered saline with Tween-20 (TBS-T) to block the aspecific antigen for $1 \mathrm{~h}$. The membrane was washed 3 times with TBS-T and probed with primary antibody at $4{ }^{\circ} \mathrm{C}$ overnight. The specific primary antibodies [APOA1, anti-apoptotic related-protein (Bcl-2), pro-apoptotic related-proteins (Bax, Caspase-3) and GAPDH] were listed in Table 2. The membrane was separated from the primary antibody and washed 3 times. The blots in membrane were probed with secondary antibody and washed 3 times. The secondary antibodies were HRP-conjugated secondary antibody (Goat Anti-Mouse, 1:2000, ab205719, Abcam, USA) and HRP-conjugated secondary antibody (Goat Anti-Rabbit, 1:2000, ab205718, Abcam, USA). BeyoECL Star (P0018FS, Beyotime) was used to visualize the target protein on the luminescent image analyzer (ImageQuant LAS4000 mini). 
Table 2

List of primary antibodies used for western blots.

\begin{tabular}{|lllll|}
\hline Protein & Antibody & $\begin{array}{l}\text { Catalog } \\
\text { Number }\end{array}$ & Company & $\begin{array}{c}\text { Antibody } \\
\text { Dilution }\end{array}$ \\
\hline APOPA1 & $\begin{array}{l}\text { Rabbit Anti-Apolipoprotein A I antibody } \\
{[\text { EP1368Y] }}\end{array}$ & ab52945 & Abcam & $1: 1000$ \\
\hline Bcl-2 & Rabbit Anti-Bcl-2 antibody (ab59348) & ab59348 & Abcam & $1: 1000$ \\
\hline Bax & Rabbit-Anti-Bax antibody [E63] & ab32503 & Abcam & $1: 1000$ \\
\hline $\begin{array}{l}\text { Cleaved } \\
\text { caspase-3 }\end{array}$ & $\begin{array}{l}\text { Rabbit Anti-Cleaved Caspase-3 antibody } \\
\text { (ab2302) }\end{array}$ & ab2302 & Abcam & $1: 1000$ \\
\hline GAPDH & $\begin{array}{l}\text { Mouse Anti-GAPDH antibody [6C5] - } \\
\text { Loading Control }\end{array}$ & ab8245 & Abcam & $1: 1000$ \\
\hline
\end{tabular}

\section{Flow Cytometry Analysis}

HBMECs were collected and stabilized with 70\% ice-cold ethanol, and dyed by Annexin V-FITC Apoptosis Detection Kit (CA1020, Solarbio) in the dark. After being washed, the apoptosis rate of BMECs was determined by flow cytometry in flow cytometer Accuri ${ }^{\text {TM }}$ C6 (BD Biosciences, USA), and the results were analyzed by Cell Quest software 3.3 (Becton-Dickinson).

\section{Scratch Wound Healing Assay}

First, BMECs $\left(5 \times 10^{5} /\right.$ well) were cultured to $80-90 \%$ confluence in 6-well plates. After the medium was discarded, confluent HBMECs were scratched using a $10 \mu$ tip, washed by serum-free DMEM, and incubated for $48 \mathrm{~h}$. The evaluation of migratory activities was performed by counting migrating cells under a $100 \times$ inverted microscope (Ts2r-FL, Nikon, Japan). Five random fields were chosen for each chamber. The following formula [( 1 - the distance following healing/the distance prior to healing) $\times$ $100 \%]$ was used to calculated relative migration rate of HBMECs.

\section{Tube Formation Assay}

Matrigel was added to the pre-cooled 24-well plates at $150 \mu \mathrm{L} /$ well. The plates were placed in an incubator at $37^{\circ} \mathrm{C}$ with $5 \% \mathrm{CO}_{2}$ for 30 min to turn into glue for next experiments. After indicated treatment for $24 \mathrm{~h}, \mathrm{HBMEC}$ were digested by trypsin and resuspended by serum-free DMEM. BMECs $\left(5 \times 10^{5} /\right.$ well) were planted into 24-well plates coated with matrigel. The $\mathrm{HC}(0.75 \mathrm{mg} / \mathrm{mL})$ was added and the cells were cultured at $37^{\circ} \mathrm{C}$ with $5 \% \mathrm{CO}_{2}$ and $95 \%$ humidity for $4 \mathrm{~h}$. Next, the cells were photographed under a $100 \times$ inverted phase contrast microscope. Three visual fields were randomly selected, and the tubule length was measured. 


\section{Statistical analysis}

The data were shown as mean \pm standard deviation (S.D.). Statistical significance in this study was analyzed by Student's $t$-test or one-way ANOVA followed by Bonferroni's post hoc test. The analyses were performed by SPSS 17.0 software (SPSS, Inc., Chicago, IL, USA). $P<0.05$ was considered statistically significant.

\section{Results}

HBMECs were identified and APOA1 participated in the protective effect of PNS on HC-inhibited cell viability

The images of HBMECs were taken under a phase-contrast microscope (Fig. 1A). The cells reached $80 \%$ confluence with fusiform or polygonal shape, and exhibited a cobblestone-like morphology. As shown in Fig. 1B, the cells highly expressed CD31 and vWF, but did not express CD133, indicating that these cells were HBMECs.

We then investigated if $\mathrm{HC}$-induced injury in HBMECs were related to the APOA1 expression. The viability of HC-injured HBMECs was determined by CCK-8 assay, and the APOA1 mRNA level was determined by qRT-PCR. As shown in Fig. $2 A$ and $B$, after treatment with various doses of $\mathrm{HC}$ for $24 \mathrm{~h}$, the cell viability was significantly reduced $(P<0.05$ or $P<0.01$ or $P<0.001)$ in parallel to a significant reduce in the mRNA level of APOA1 $(P<0.01$ or $P<0.001)$. We detected the effect of PNS on cell viability and the mRNA level of APOA1 in HBMECs. As shown in Fig. $2 \mathrm{C}$ and $\mathrm{D}$, after treatment with various doses of PNS for $24 \mathrm{~h}$, the cell viability was not changed significantly while the mRNA level of APOA1 was significantly increased ( $P$ $<0.05$ or $\mathrm{P}<0.01$ or $\mathrm{P}<0.001$ ). We explored whether $\mathrm{PNS}$ had a protective effect on $\mathrm{HC}$-induced injury in HBMECs. As exhibited in Fig. 2E and F, PNS at the concentration range of $300-400 \mathrm{mg} / \mathrm{ml}$ significantly reversed part of the $\mathrm{HC}$-induced cell injury $(\mathrm{P}<0.05$ or $\mathrm{P}<0.01)$, and $\mathrm{PNS}$ at the concentration range of $200-400 \mathrm{mg} / \mathrm{ml}$ significantly increased the mRNA level of APOA1 $(\mathrm{P}<0.05$ or $\mathrm{P}<0.001)$. Moreover, the effects of PNS on HC-induced injury in HBMECs and APOA1 mRNA level showed a dose-dependent manner; PNS (200-400 mg/ml) did not obviously change the viability of normal HBMECs, so we chose PNS (400 mg/ml) for next experiments. According to the above results, we speculated that APOA1 expression may be involved in the preventing effect of PNS on HC-induced injury in HBMECs.

\section{APOA1 was involved in the preventing effect of PNS on HC-induced injury in HBMECs}

As shown in Fig. 3A and B, both protein and mRNA levels of APOA1 were significantly increased after APOA1 transfection $(P<0.001)$, while both of them were significantly decreased after siAPOA1 transfection $(P<0.001)$. We examined whether PNS and over-expressed APOA1 had a synergistic protective effect on $\mathrm{HC}$-induced injury in HBMECs. As shown in Fig. $3 \mathrm{C}$, relative to $\mathrm{HC}$ alone, the combination of $\mathrm{HC}$ and siAPOA1 further promoted cell injury $(P<0.001)$, but PNS treatment reversed this effect of siAPOA1 $(P<0.01)$. Relative to $\mathrm{HC}$ alone, the combination of PNS and $\mathrm{HC}$ treatment or APOA1 
and $\mathrm{HC}$ treatment inhibited cell injury $(\mathrm{P}<0.01)$; the effect of PNS was further enhanced by APOA1 treatment $(P<0.05)$, and the effect of APOA1 was further enhanced by PNS treatment $(P<0.05)$.

\section{APOA1 was involved in the preventing effect of PNS on HC-induced apoptosis in HBMECs}

The effects of APOA1, siAPOA1 and PNS treatment on HC-induced apoptosis in HBMECs were determined by flow cytometry and Western blot assays. According to Fig. 4A, relative to control group, HC led to a significantly enhanced apoptotic rate in HBMECs $(P<0.001)$ and the combination of $\mathrm{HC}$ and siAPOA1 further promoted the cell apoptotic rate $(P<0.001)$, while PNS treatment reversed this effect of siAPOA1 $(P<0.001)$. By contrast, PNS treatment or APOA1 treatment attenuated the HC-induced apoptosis $(P<0.001)$; the combination treatment of PNS and APOA1 further significantly attenuated the HC-induced apoptosis $(\mathrm{P}<0.001)$; likewise, siAPOA1 treatment partially reversed this effect of PNS $(\mathrm{P}<$ 0.05). Cell injury is generally associated with changes in the expressions of apoptosis related-proteins. According to Fig. 4B, compared with control group, $\mathrm{HC}$ obviously decreased the protein level of $\mathrm{Bcl}-2$ and increased the protein levels of Bax and Caspase- 3 in HBMECs; the combination of HC and siAPOA1 further decreased the protein level of $\mathrm{Bcl}-2$ and increased the protein levels of Bax and Caspase-3; however, PNS treatment reversed this effect of siAPOA1. Conversely, PNS or APOA1 increased the protein level of Bcl-2 and decreased the protein levels of Bax and Caspase-3, and the combination treatment of PNS and APOA1 further significantly promoted the protein level of $\mathrm{Bcl}-2$ and suppressed the protein levels of Bax and Caspase-3.

\section{APOA1 was involved in the preventing effect of PNS on HC-inhibited migration and angiogenesis in HBMECs}

According to Fig. 5A, compared with control group, $\mathrm{HC}$ significantly suppressed the migration rate in HBMECs $(\mathrm{P}<0.001)$, and the combination of $\mathrm{HC}$ and siAPOA1 further suppressed the migration rate $(\mathrm{P}<$ 0.001); however, PNS treatment reversed this effect of siAPOA1 $(P<0.001)$. By contrast, PNS or APOA1 treatment promoted the migration rate $(P<0.001)$, and the combination treatment of PNS and APOA1 further significantly promoted the migration rate $(P<0.001)$; likewise, siAPOA1 treatment reversed this effect of PNS $(P<0.001)$.

According to Fig. 5B, compared with control group, $\mathrm{HC}$ significantly suppressed the tube length in HBMECs $(P<0.01)$ and the combination of $\mathrm{HC}$ and siAPOA1 further suppressed the tube length $(\mathrm{P}<$ 0.001); however, PNS treatment reversed this effect of siAPOA1 $(P<0.001)$. By contrast, PNS or APOA1 treatment promoted the tube length $(P<0.05)$, and the combination treatment of PNS and APOA1 further obviously promoted the tube length $(P<0.01)$; likewise, siAPOA1 treatment reversed this effect of PNS $(P$ $<0.001)$. Thus, the data suggested that the changes of APOA1 expression by APOA1 or siAPOA1 transfection were involved in the preventing effect of PNS on $\mathrm{HC}$-inhibited migration and angiogenesis in HBMECs.

\section{Discussion}


ONFH is still a disabling condition with ill-defined etiology and pathogenesis (18). High levels of glucocorticoid-induced ONFH were observed in patients who underwent total hip replacement (19). We therefore used $\mathrm{HC}$ to injure HBMECs to establish a glucocorticoid-induced ONFH in vitro model and explored the protective role of PNS in HC-induced injury in HBMECs. This study may introduce PNS as a novel treatment strategy on ONFH.

Accumulated evidence indicates that PNS has protective effect on various injured cells. PNS exerts protective effects against high glucose-induced oxidative injury in rat retinal capillary endothelial cells (13). PNS also attenuated the inhibitory effect of doxorubicin-induced damage on H9C2 cells (20). In this study, we found that PNS could attenuate the inhibitory effect of HC on the viability of HBMECs, implying PNS had a protective effect on HC-induced injury in HBMECs, which suggested that PNS had potential therapeutic effect on ONFH. More importantly, HC decreased the expression of APOA1 in a dosedependent manner, and PNS increased the expression of APOA1 in the same manner. We therefore suggested that the protective effect of PNS on HC-induced injury in HBMECs was related with the differential expression of APOA1. To further investigate the relationship between PNS and APOA1 in the protective effect on $\mathrm{HC}$-induced cell injury, we transfected HBMECs with APOA1 or siAPOA1 to up-regulate or down-regulate APOA1 expression. The results revealed that PNS protected HBMECs against HCinduced injury through promoting the expression of APOA1. When the expression of APOA1 was suppressed, the protective effect of PNS on HC-injured HBMECs was also suppressed.

Previous studies have shown that apoptosis is induced by high concentration of glucocorticoids in osteocytes (21). Apoptosis is one type of programed cell death and plays a significant role in the loss of microvascular function (22). Apoptosis is modulated by many factors, especially Bcl-2 (inhibitors) and Bax (promoters), and by activation of the apoptotic cascade via caspase-3, the protein cleaved (activated) by which is considered as an executioner of apoptotic pathway and requires induction of apoptosis (23). In this study, we found that HC or APOA1 knockdown not only increased the apoptotic rate, but also inhibited the expression of Bcl-2 and promoted the expressions of Bax and Caspase-3. APOA1 knockdown further decreased HC-induced apoptosis; PNS inhibited apoptosis and reversed this effect of APOA1 knockdown; PNS and APOA1 over-expressions further significantly inhibited apoptosis. We demonstrated that PNS can protect HBMECs in HC condition by inhibiting apoptosis, and this effect of PNS was involved in the promotion of APOA1. Numerous studies have suggested PNS has antiapoptotic effect. For example, PNS attenuates cardiomyocyte apoptosis through mitochondrial pathway in natural aging rats (24). PNS protects against SH-SY5Y cell apoptosis induced by 6-hydroxydopamine (25) and K562 cell apoptosis (26). Besides, total PNS also exerts anti-apoptotic effect on rat bone marrow mesenchymal stem cells (27).

The migration of vascular endothelial cells is not only the key step of angiogenesis, but also the basis of neovascularization (28). Angiogenesis, the growth of new blood vessels from existing vessels, is an important aspect of the repair process of vascular injury (29). To further elucidate the role of PNS and APOA1 in HC-inhibited migration and tube formation of HBMECs, we co-cultured PNS with HBMECs exposed to $\mathrm{HC}$ condition. The results suggested that PNS treatment can promote the migration and tube 
formation of HBMECs through increasing the expression of APOA1, implying PNS promotes angiogenesis of $\mathrm{HC}$-induced $\mathrm{ONFH}$, and the elucidation of this mechanism may contribute to the treatment of ONFH. As for cancer, however, PNS inhibits migration in mouse breast carcinoma cell line (30); similar reports also showed that total PNS inhibits migration of human prostate cancer PC-3 cells (31). Additionally, apart from the effect on angiogenesis of HBMECs, PNS also promotes angiogenesis through AMPK and eNOS dependent pathways in HUVECs (32); PNS could enhance angiogenesis and the pro-angiogenic effects through the VEGF-KDR/Flk-1 and PI3K-Akt-eNOS signaling pathways in vivo (HUVECs) and in vitro (zebrafish), respectively (33). Similar results reported that total PNS promotes angiogenesis deriving from rat bone marrow mesenchymal stem cells (34). By contrast, PNS suppresses angiogenesis of atherosclerotic plaque by decreasing vascular endothelial growth factors and the expression of nicotinamide adenine dinucleotide phosphate oxidase subunit 4 (35).

Although our research illustrated the mechanism underlying PNS on cell viability, migration and tube formation in HC-injured HBMECs, more studies should be performed in appropriate animal models to further investigate clinical application of PNS on HC-injured ONFH. These attractive data revealed that the strategy that PNS promotes AOPA1 expression to protect $\mathrm{HC}$-injured HBMECs might provide a promising therapeutic method for $\mathrm{HC}$-injured ONFH.

\section{Conclusions}

Taken together, we demonstrated that PNS prevents HC-induced injury in HBMECs, and changes of APOA1 expression are crucial throughout the whole process. $\mathrm{HC}$ inhibits the APOA1 expression, while PNS promotes the APOA1 expression. PNS protect $\mathrm{HC}$-injured HBMECs through promoting the expression of APOA1. Further, PNS and over-expressed APOA1 had a synergistic protective effect on $\mathrm{HC}$-induced injury in HBMECs. Our results might provide evidence to support PNS use in the treatment of ONFH.

\section{Abbreviations}

PNS

panax notoginseng saponins

$\mathrm{HC}$

hydrocortisone

HBMECs

human bone microvascular endothelial cells

ONFH

Osteonecrosis of the femoral head

BMECs

bone microvascular endothelial cells

APOA1

Apolipoprotein A1

HDL 
high-density lipoprotein

\section{Declarations}

\section{Ethics approval and consent to participate}

All procedures performed in studies involving human participants were in accordance with the ethical standards of the institutional and/or national research committee and with the 1964 Helsinki declaration and its later amendments or comparable ethical standards.

No human or animals are involved in this research.

\section{Availiability of Data and Materials}

The analysed data sets generated during the study are available from the corresponding author on reasonable request.

\section{Consent for publication}

Not applicable.

\section{Availiability of Data and Materials}

The analysed data sets generated during the study are available from the corresponding author on reasonable request.

\section{Disclosure of Conflict-of-Interest}

The authors declare no conflicts of interest.

\section{Funding}

Not applicable

\section{Authors' contributions}

Substantial contributions to conception and design: JJ, YF

Data acquisition, data analysis and interpretation:ST, WG,BS, LY, LB, ML

Drafting the article or critically revising it for important intellectual content: JJ, YF

Final approval of the version to be published: All authors

Agreement to be accountable for all aspects of the work in ensuring that questions related to the accuracy or integrity of the work are appropriately investigated and resolved 
Acknowledgements

Not applicable

\section{References}

1. Cohen-Rosenblum A, Cui Q. Osteonecrosis of the Femoral Head. The Orthopedic clinics of North America. 2019;50(2):139-49.

2. Liu LH, Zhang QY, Sun W, Li ZR, Gao FQ. Corticosteroid-induced Osteonecrosis of the Femoral Head: Detection, Diagnosis, and Treatment in Earlier Stages. Chin Med J. 2017;130(21):2601-7.

3. Yu QS, Guo WS, Cheng LM, Lu YF, Shen JY, Li P. Glucocorticoids Significantly Influence the Transcriptome of Bone Microvascular Endothelial Cells of Human Femoral Head. Chin Med J. 2015;128(14):1956-63.

4. Zhang Q, Gao F, Cheng L, Liu L, Sun W, Li Z. [Effects of icariin on autophagy and exosome production of bone microvascular endothelial cells]. Zhongguo xiu fu chong jian wai ke za zhi = Zhongguo xiufu chongjian waike zazhi $=$ Chinese journal of reparative and reconstructive surgery. 2019;33(5):56877.

5. Kang P, Shen B, Yang J, Pei F. Circulating platelet-derived microparticles and endothelium-derived microparticles may be a potential cause of microthrombosis in patients with osteonecrosis of the femoral head. Thrombosis research. 2008;123(2):367-73.

6. Gan C, Wang Z, Chen Y. In situ AFM imaging of apolipoprotein A-I directly derived from plasma HDL. Atherosclerosis. 2017;259:5-11.

7. Chistiakov DA, Orekhov AN, Bobryshev YV. ApoA1 and ApoA1-specific self-antibodies in cardiovascular disease. Lab Invest. 2016;96(7):708-18.

8. Sengupta MB, Saha S, Mohanty PK, Mukhopadhyay KK, Mukhopadhyay D. Increased expression of ApoA1 after neuronal injury may be beneficial for healing. Molecular cellular biochemistry. 2017;424(1-2):45-55.

9. Wu X, Yu Z, Su W, Isquith DA, Neradilek MB, Lu N, et al. Low levels of ApoA1 improve risk prediction of type 2 diabetes mellitus. J Clin Lipidol. 2017;11(2):362-8.

10. Tuft Stavnes H, Nymoen DA, Hetland Falkenthal TE, Kaern J, Trope CG, Davidson B. APOA1 mRNA expression in ovarian serous carcinoma effusions is a marker of longer survival. Am J Clin Pathol. 2014;142(1):51-7.

11. Layne K, Ferro A. Traditional Chinese medicines in the management of cardiovascular diseases: a comprehensive systematic review. Br J Clin Pharmacol. 2017;83(1):20-32.

12. Uzayisenga R, Ayeka PA, Wang Y. Anti-diabetic potential of Panax notoginseng saponins (PNS): a review. Phytother Res. 2014;28(4):510-6.

13. Fan Y, Qiao Y, Huang J, Tang M. Protective Effects of Panax notoginseng Saponins against High Glucose-Induced Oxidative Injury in Rat Retinal Capillary Endothelial Cells. Evidence-based 
complementary and alternative medicine: eCAM. 2016;2016:5326382.

14. Yue J, Wan F, Zhang Q, Wen P, Cheng L, Li P, et al. Effect of glucocorticoids on miRNA expression spectrum of rat femoral head microcirculation endothelial cells. Gene. 2018;651:126-33.

15. Janeczek Portalska K, Leferink A, Groen N, Fernandes H, Moroni L, van Blitterswijk C, et al. Endothelial differentiation of mesenchymal stromal cells. PloS one. 2012;7(10):e46842.

16. Wang X, Zhang G, Zhu C, Lin L, Zhao Z, Yu X, et al. Vitamin C Prevents Hydrocortisone-Induced Injury in HMEC-1 through Promoting Bestrophin-3 Expression. Nutrition cancer. 2019;71(5):852-60.

17. D.Schmittgen JLivakK. T. Analysis of relative gene expression using different real-time quantitative PCR and 2- $\triangle \triangle C T$ method. Acta Agronomica Sinica. 2001(25):402-8.

18. Tripathy SK, Goyal T, Sen RK. Management of femoral head osteonecrosis: Current concepts. Indian journal of orthopaedics. 2015;49(1):28-45.

19. Moya-Angeler J, Gianakos AL, Villa JC, Ni A, Lane JM. Current concepts on osteonecrosis of the femoral head. World journal of orthopedics. 2015;6(8):590-601.

20. Shi R, Liu L, Huo Y, Cheng YY. [Study on protective effects of Panax notoginseng saponins on doxorubicin-induced myocardial damage]. Zhongguo Zhong yao za zhi = Zhongguo zhongyao zazhi $=$ China. journal of Chinese materia medica. 2007;32(24):2632-5.

21. Jia J, Yao W, Guan M, Dai W, Shahnazari M, Kar R, et al. Glucocorticoid dose determines osteocyte cell fate. FASEB journal: official publication of the Federation of American Societies for Experimental Biology. 2011;25(10):3366-76.

22. Zhu G, Wang X, Wu S, Li X, Li Q. Neuroprotective effects of puerarin on 1-methyl-4-phenyl-1,2,3,6tetrahydropyridine induced Parkinson's disease model in mice. Phytother Res. 2014;28(2):179-86.

23. Dolka I, Krol M, Sapierzynski R. Evaluation of apoptosis-associated protein (Bcl-2, Bax, cleaved caspase-3 and p53) expression in canine mammary tumors: An immunohistochemical and prognostic study. Res Vet Sci. 2016;105:124-33.

24. Zhou Z, Wang J, Song Y, He Y, Zhang C, Liu C, et al. Panax notoginseng saponins attenuate cardiomyocyte apoptosis through mitochondrial pathway in natural aging rats. Phytother Res. 2018;32(2):243-50.

25. Wang MX, Zhao JY, Sun DM, Meng XB, Sun GB, Sun XB. [Protective effects and underlying mechanisms of Panax notoginseng saponins against SH-SY5Y cell apoptosis induced by 6hydroxydopamine]. Yao xue xue bao = Acta pharmaceutica Sinica. 2016;51(6):898-906.

26. Li Y, Zhai W, Yang X, Ding J, Kan L. [Effects of Panax notoginseng saponins on proliferation, apoptosis and cell cycle of K562 cells in vitro and the mechanisms]. Nan fang yi ke da xue xue bao = Journal of Southern Medical University. 2015;35(8):1103-9.

27. Ou YF, Fu XK, Mei XS, Zheng HZ. [Inhibitory effect of total saponins of Panax notoginseng on rat bone marrow mesenchymal stem cell apoptosis]. Sheng Li Xue Bao. 2016;68(3):285-92.

28. Treggiari D, Dalbeni A, Meneguzzi A, Delva P, Fava C, Molesini B, et al. Lycopene inhibits endothelial cells migration induced by vascular endothelial growth factor $A$ increasing nitric oxide bioavailability. 
J Funct Foods. 2018;42:312-8.

29. Johnson KE, Wilgus TA. Vascular Endothelial Growth Factor and Angiogenesis in the Regulation of Cutaneous Wound Repair. Advances in wound care. 2014;3(10):647-61.

30. Wang P, Cui J, Du X, Yang Q, Jia C, Xiong M, et al. Panax notoginseng saponins (PNS) inhibits breast cancer metastasis. J Ethnopharmacol. 2014;154(3):663-71.

31. Xiaochen NI, Zhao Z, Wei Q. Inhibition of the Proliferation and Migration of Human Prostate Cancer PC-3 Cells by Total Panax Notoginseng Saponins. Chinese Journal of Clinical Oncology. 2011;38(11):638-41.

32. Wang D, Jie Q, Liu B, Li Y, Dai L, Luo J, et al. Saponin extract from Panax notoginseng promotesangiogenesis through AMPK and eNOSdependent pathways in HUVECs. Mol Med Rep. 2017;16(4):5211-8.

33. Hong SJ, Wan JB, Zhang Y, Hu G, Lin HC, Seto SW, et al. Angiogenic effect of saponin extract from Panax notoginseng on HUVECs in vitro and zebrafish in vivo. Phytother Res. 2009;23(5):677-86.

34. Zheng H, Liu C, Ou Y, Zhang Y, Fu X. Total saponins of Panax notoginseng enhance VEGF and relative receptors signals and promote angiogenesis derived from rat bone marrow mesenchymal stem cells. J Ethnopharmacol. 2013;147(3):595-602.

35. Qiao Y, Zhang PJ, Lu XT, Sun WW, Liu GL, Ren M, et al. Panax notoginseng saponins inhibits atherosclerotic plaque angiogenesis by down-regulating vascular endothelial growth factor and nicotinamide adenine dinucleotide phosphate oxidase subunit 4 expression. Chin J Integr Med. 2015;21(4):259-65.

\section{Figures}



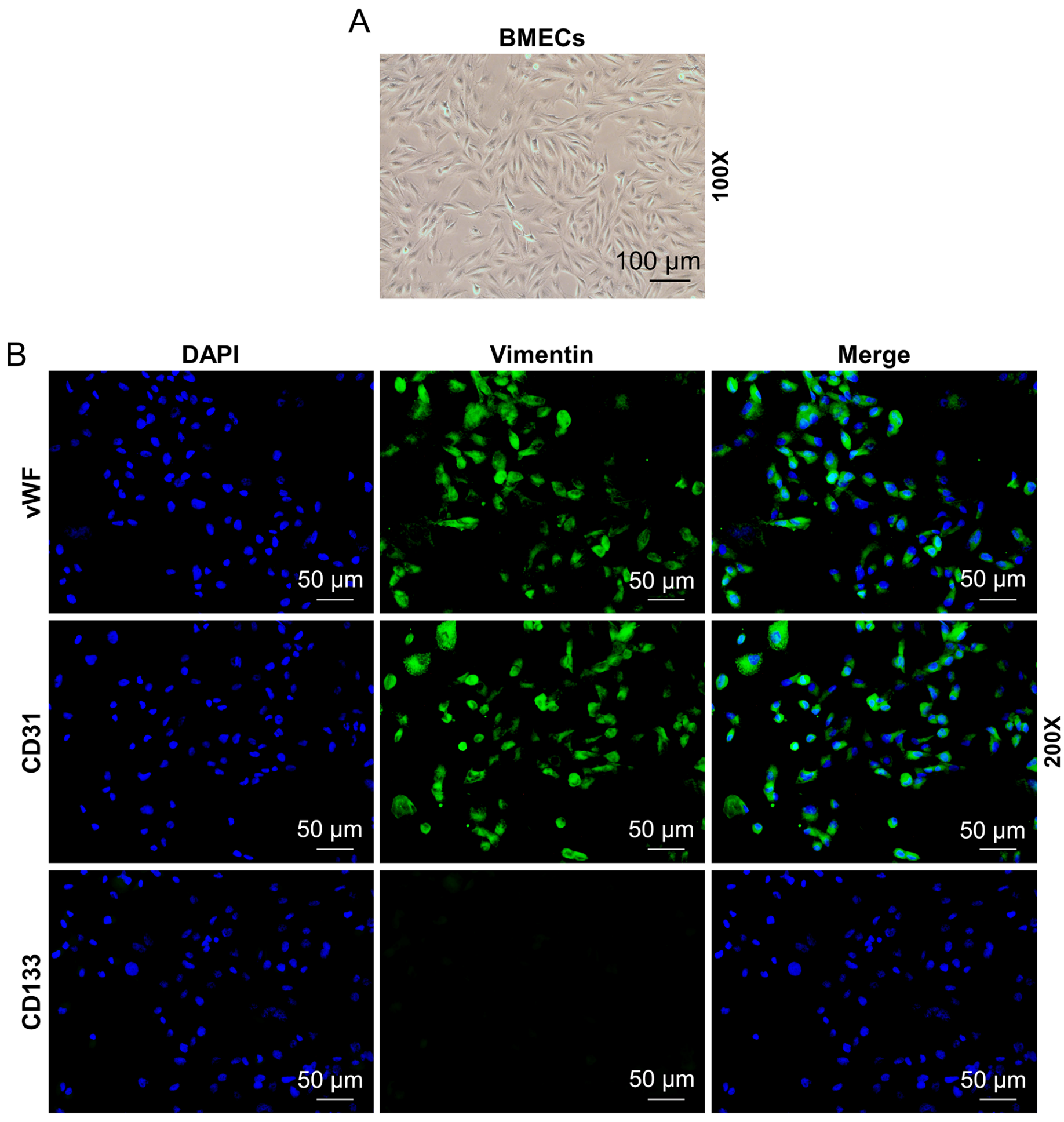

Figure 2

Identification of human bone microvascular endothelial cells (HMECs). (A) Initial BMECs morphology (100x magnification). (B) Cells highly expressed vWF and CD31, whereas were negative for CD133 (100x magnification). 
A

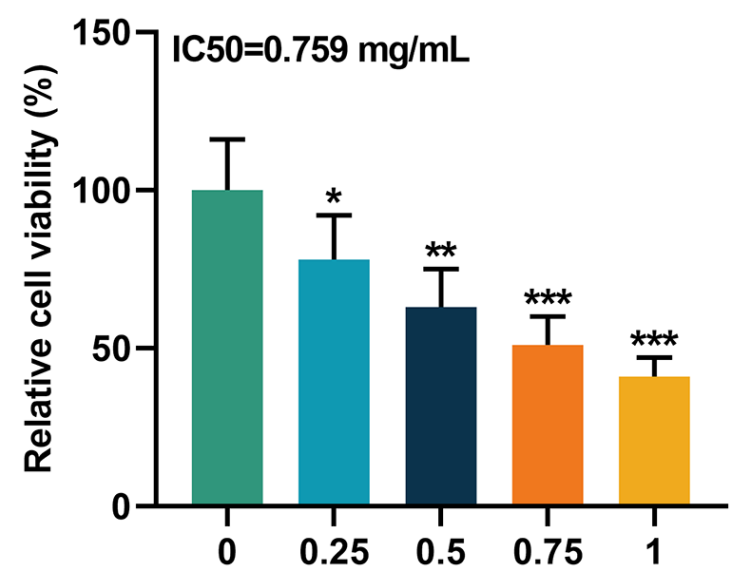

C

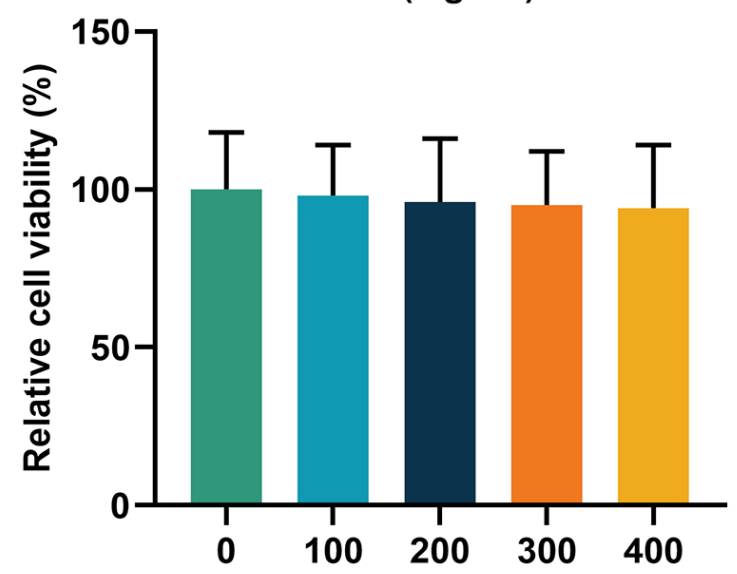

$E$

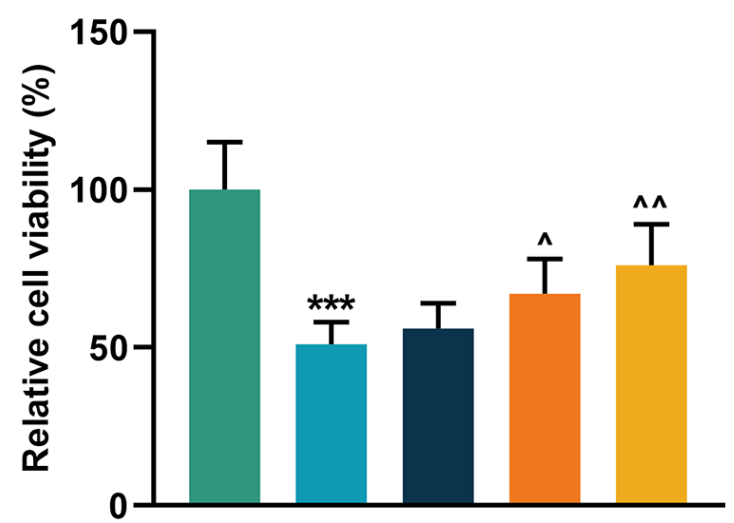

$\begin{array}{llllll}\mathrm{HC}(\mathrm{mg} / \mathrm{mL}) & 0 & 0.75 & 0.75 & 0.75 & 0.75\end{array}$

$\begin{array}{llllll}\text { PNS }(\mathrm{mg} / \mathrm{mL}) & 0 & 0 & 200 & 300 & 400\end{array}$
B

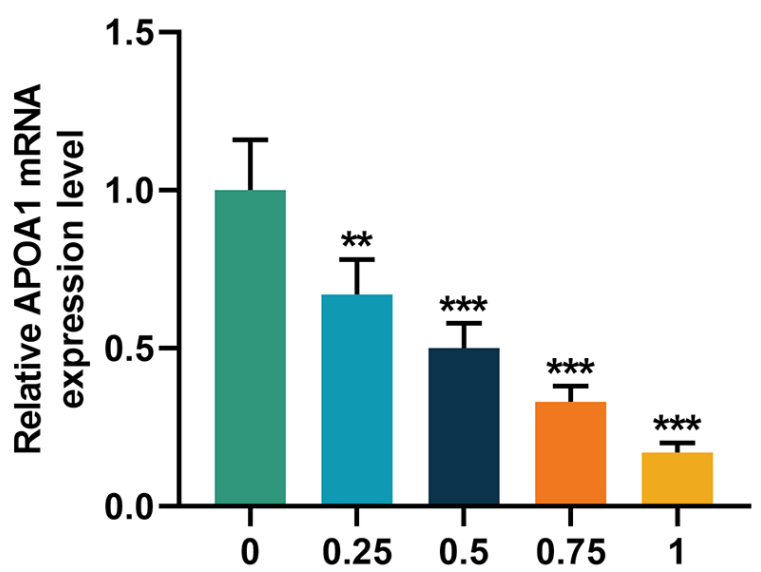

$\mathrm{HC}(\mathrm{mg} / \mathrm{mL})$

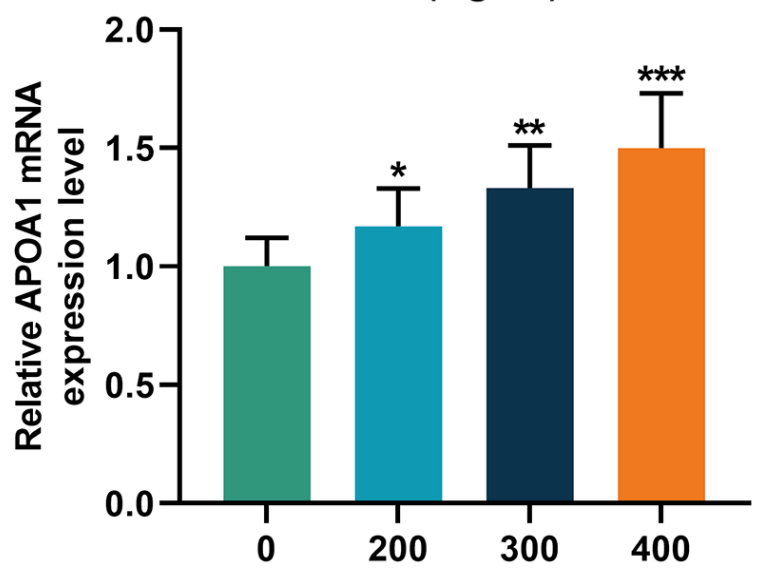

F

PNS (mg/mL)

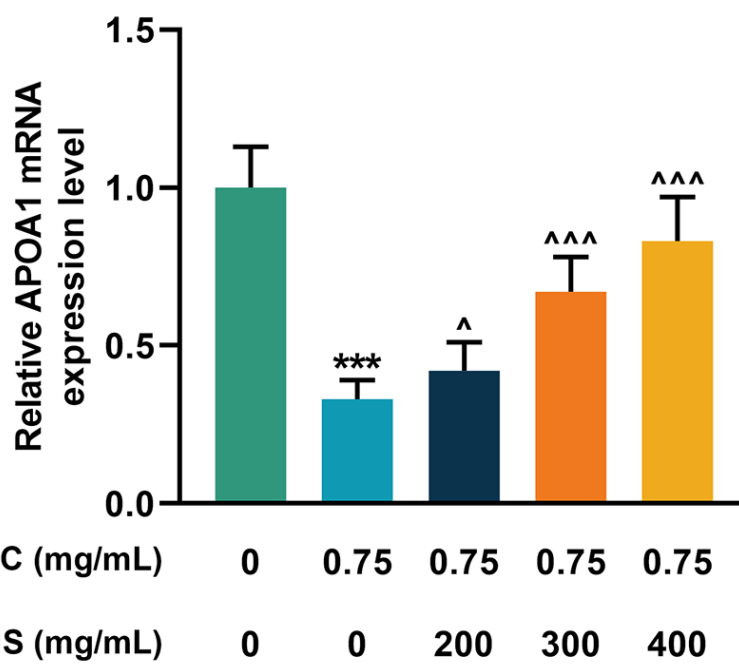

Figure 4

APOA1 is involved in the effect of PNS on the viability of HC-induced BMECs. (A) Hydrocortisone (HC) inhibits BMECs viability in a dose-dependent manner. (B) HC inhibits mRNA expression of APOA1 in a dose-dependent manner. (C) The BMECs viability is treated with panax notoginseng saponins (PNS). (D) PNS promotes mRNA expression of APOA1 in a dose-dependent manner. (E) PNS prevents HC inhibited the viability of BMECs. (F) PNS promotes BMECs viability in parallel to higher expression of APOA1 in a 
dose-dependent manner. ${ }^{\star} \mathrm{P}<0.05$ or ${ }^{\star \star} \mathrm{P}<0.01$ or ${ }^{\star \star *} \mathrm{P}<0.001 \mathrm{vs}$. $0 \mathrm{mg} / \mathrm{mL} .{ }^{\wedge} \mathrm{P}<0.05$ or ${ }^{\wedge} \mathrm{P}<0.01$ or ${ }^{\wedge \wedge \wedge} \mathrm{P}<0.001$ vs. HC. The data were shown as mean \pm standard deviation (S.D.).
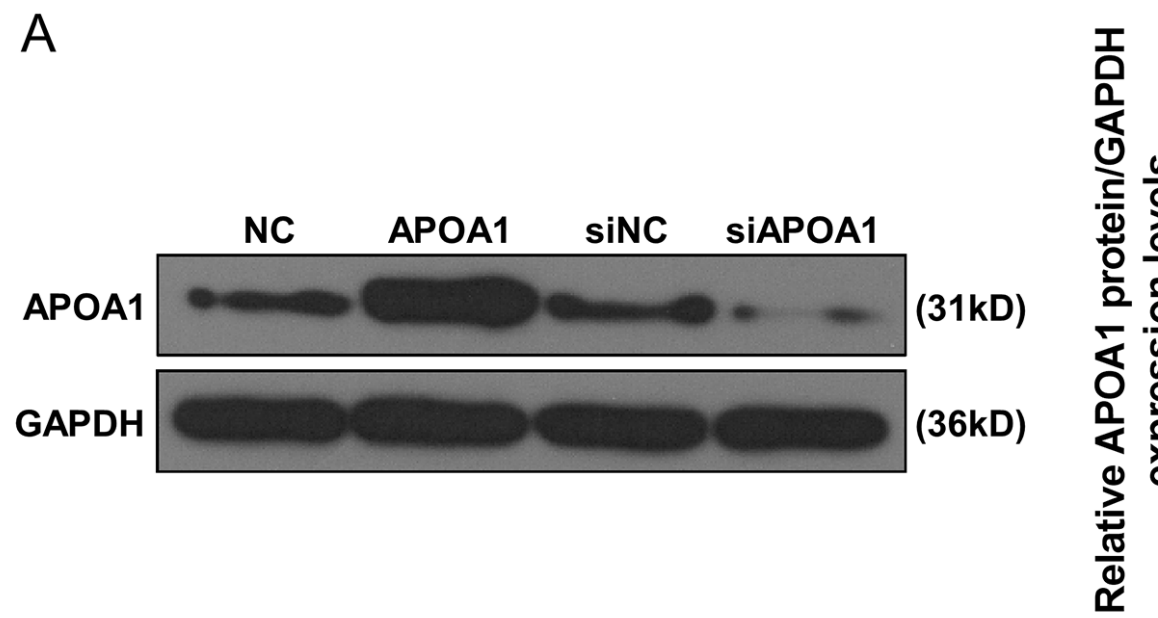

$(31 \mathrm{kD})$

$(36 \mathrm{kD})$

B

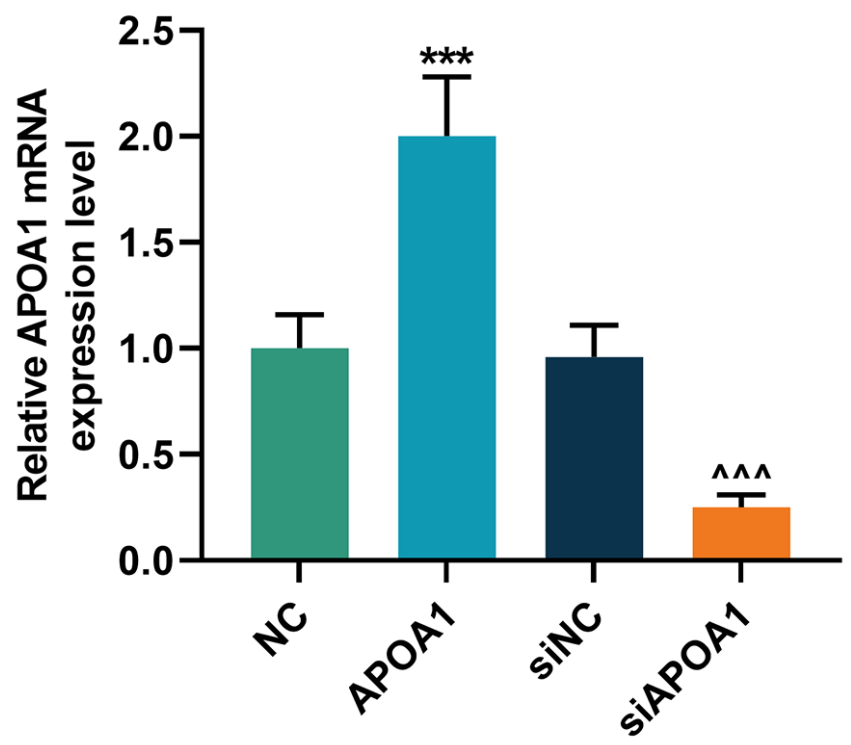

C

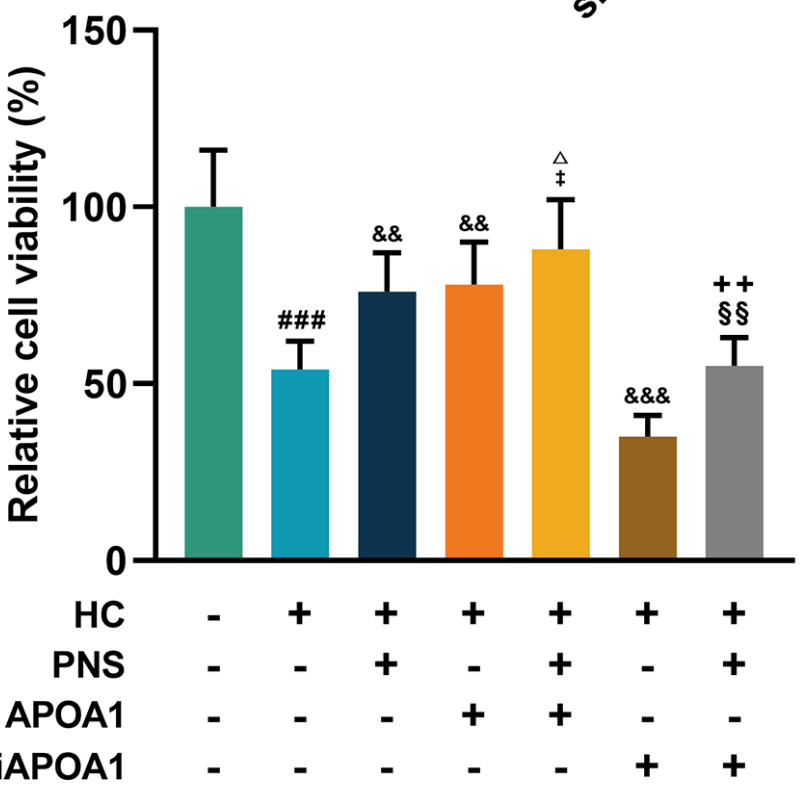

Figure 5

The effects of APOA1 expression on PNS preventing hydrocortisone (HC)-injured BMECs. (A) Protein level of APOA1 was altered by transfection of APOA1 or APOA1 siRNA (siAPOA1) in BMECs. (B) mRNA level of APOA1 was altered by transfection of APOA1 or APOA1 siRNA (siAPOA1) in BMECs. (C) Effects of altered APOA1 expression by transfection with APOA1 or siAPOA1 on PNS preventing HC-induced injury in BMECs. ${ }^{* \star *} \mathrm{P}<0.001$ vs. negative control (NC). ${ }^{\wedge \wedge} \mathrm{P}<0.001$ vs. siRNA negative control (siNC). \#\#\#P $<0.001$ vs. Control. \&\&P $<0.05$ or $\& \& \& P<0.001$ vs. $H C . \triangle P<0.05$ vs. $H C+P N S$. $\ddagger P<0.05$ vs. $H C+A P O A 1$. $++\mathrm{P}<0.01$ vs. $\mathrm{HC}+\mathrm{PNS}$. $\S \S \mathrm{P}<0.01$ vs. $\mathrm{HC}+$ siAPOA1. The data were shown as mean \pm standard deviation (S.D.). 
A
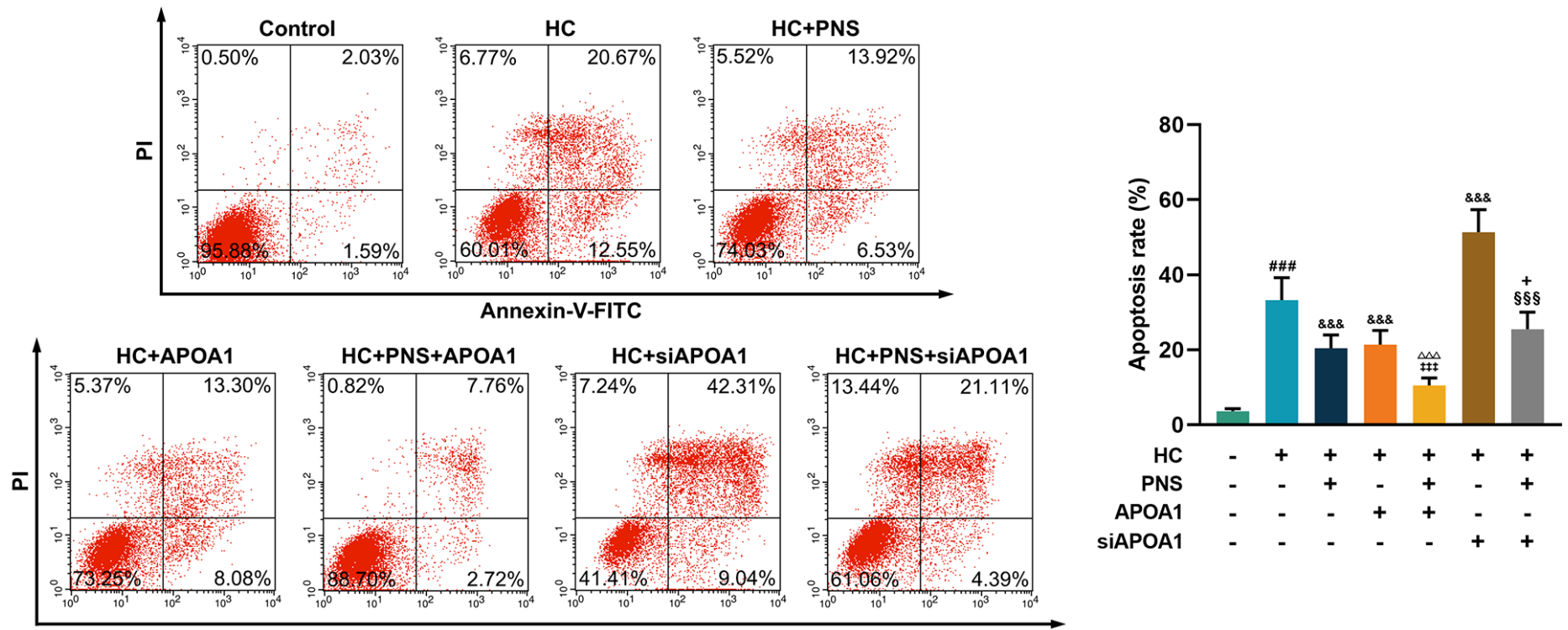

Annexin-V-FITC
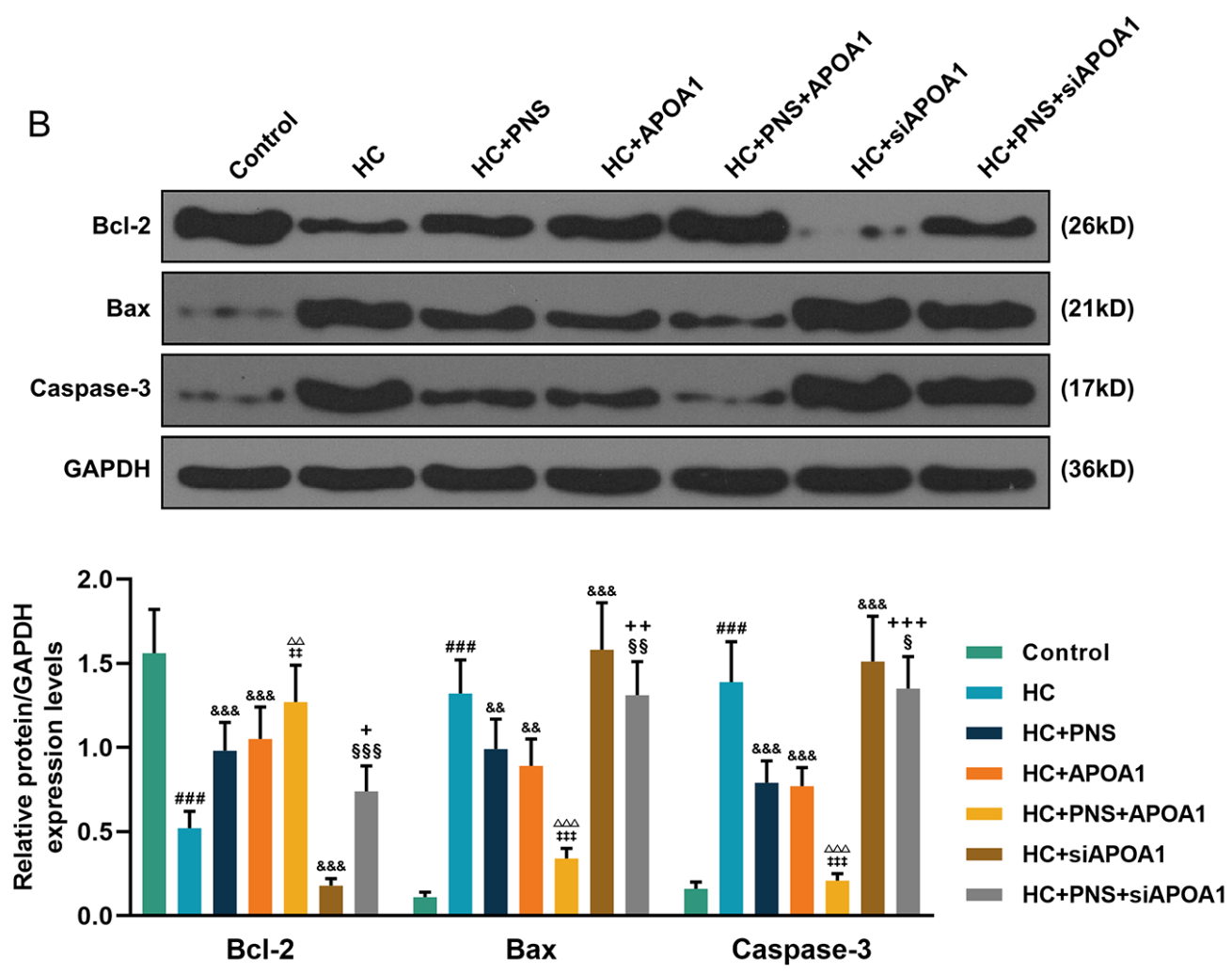

\section{Figure 7}

The effects of APOA1 expression on PNS preventing hydrocortisone (HC)-indured apoptosis in BMECs. (A) Effects of altered APOA1 expression by transfection with APOA1 or siAPOA1 on PNS preventing HCinduced apoptosis in BMECs. (B) Effects of altered APOA1 expression by transfection with APOA1 or siAPOA1 on PNS reversing HC-induced apoptotsis-related proteins expression in BMECs. \#\#\#P <0.001 vs.

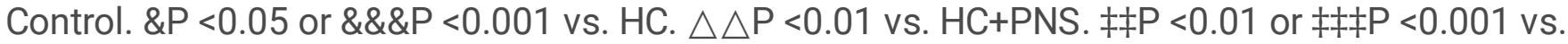
HC+APOA1. $+\mathrm{P}<0.05$ or $++\mathrm{P}<0.01$ or $+++\mathrm{P}<0.001$ vs. HC+PNS. $\S \mathrm{P}<0.05$ or $\S \S \S \mathrm{P}<0.001$ vs. HC+siAPOA1. The data were shown as mean \pm standard deviation (S.D.). 
A
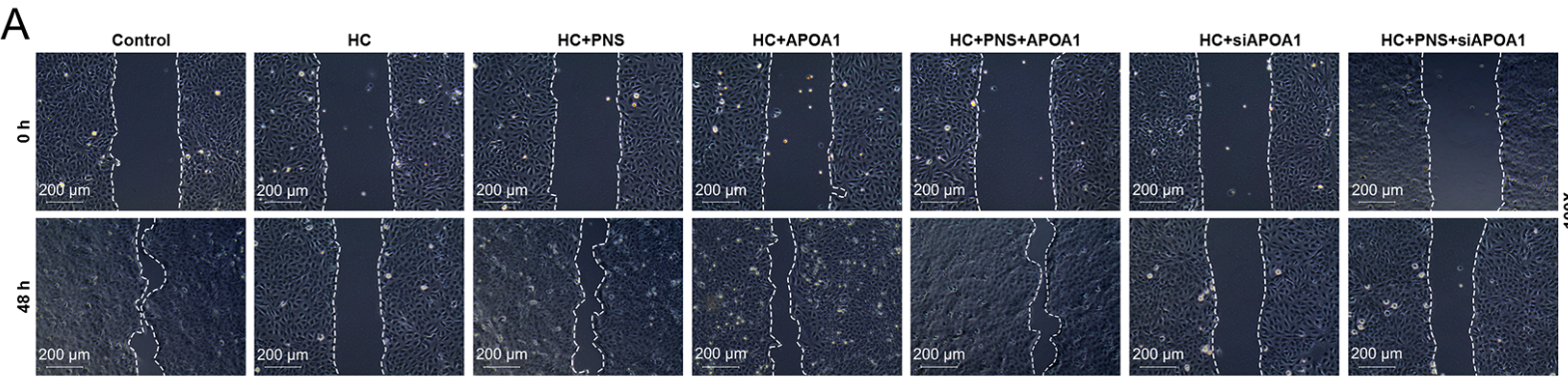

B

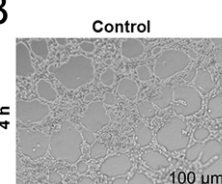

$\mathrm{HC}$

HC+PNS

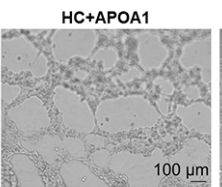

HC+PNS+APOA1

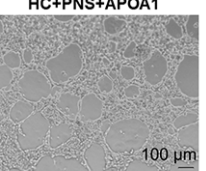

HC+SIAPOA1

HC+PNS+SIAPOA

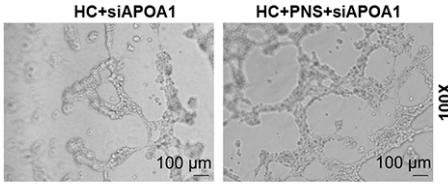

Figure 10

The effects of APOA1 expression on PNS preventing hydrocortisone (HC)-inhibited migration and tube formation in BMECs. (A) Scratch test to detect effects of altered APOA1 expression by transfection with APOA1 or siAPOA1 on PNS preventing HC-inhibited migration in BMECs. (Inverted phase contrast microscopex 100) (B) Tube formation assay to deter the effects of altered APOA1 expression by transfection with APOA1 or siAPOA1 on PNS preventing HC-inhibited tube length in BMECs. (Inverted phase contrast microscope 100$)$. \#\#P $<0.01$ or \#\#\#P $<0.001$ vs. Control. $\& P<0.05$ or $\& \& \& P<0.001$ vs. HC. $\triangle \triangle P<0.01$ or $\triangle \triangle \triangle P<0.01$ vs. HC+PNS. $\ddagger \ddagger P<0.01$ or $\ddagger \ddagger \ddagger P<0.001$ vs. $H C+A P O A 1 .+++P<0.001$ vs. $\mathrm{HC}+\mathrm{PNS}$. $\S \S \S \mathrm{P}<0.001$ vs. $\mathrm{HC}+$ siAPOA1. The data were shown as mean \pm standard deviation (S.D.). 